\title{
EDUCATION
}

\section{WIMS, a Community of Teachers, Developers and Users}

\section{Marina Cazzola, Sophie Lemaire, and Bernadette Perrin-Riou}

\section{Introduction}

The so-called new technologies are nowadays pervasive in every aspect of our lives, and their use in communication of science and education is an established need, made even more evident by the COVID-19 emergency. The aims for such use can be various. On one hand, in the spirit of improving teaching, computers can be used to improve the quality of the materials offered to students, to the extent allowed by the new media. From a completely different point of view, the use of computerized tests can have the effect of reducing teachers' workload, so as to save energies that then can be directed towards tasks more significant than just marking. Furthermore the possibility of building personalized environments could put the students in a position to work at their own pace and develop self-directed learning skills.

Having been engaged in the development of WIMS (WWW Interactive Multipurpose Server) for almost two decades, we wish to contribute an analysis of our experience with the benefits that these technologies can bring.

Marina Cazzola is a researcher and lecturer in mathematics education at Dipartimento di Matematica e Applicazioni, Università degli Studi di MilanoBicocca, Milano, Italia. Her email address is marina. cazzola@unimib. it. Sophie Lemaire is a researcher and lecturer in mathematics at Université ParisSaclay, CNRS, Laboratoire de mathématiques d'Orsay, France. Her email address is sophie.1emaire@universite-paris-saclay.fr.

Bernadette Perrin-Riou is an emeritus professor in mathematics at Université Paris-Saclay, CNRS, Laboratoire de mathématiques d'Orsay, France. Her email address is bernadette. perrin-riou@universite-paris-saclay. fr.

For permission to reprint this article, please contact:

reprint-permission@ams.org.

\section{About WIMS}

The WIMS system started as an individual project of Xiao Gang, based at Département de mathématiques at Université de Nice - Sophia Antipolis (France), and had been made available as open source in 1998 [14]. Xiao's project aimed at "a systematic approach for providing Internet-accessible mathematical computations" ([14]). It included, since the beginning, basic LMS-like functionalities: virtual classes for teacher to dispense learning materials to the students and monitor their achievements, a grading system, a forum, .... Nowadays, under the name WIMS you can find a network of servers sharing interactive resources at many levels in various subjects (not only mathematics but also biology, chemistry, economics, languages, physics, ...), most of which have automatic correction and marking of user input. The LMS functionalities in WIMS have significantly evolved since the beginning (better user management, better feedback for users' inputs, e.g., through opportune links to specific documents compiled by the teachers). WIMS also provides a secure exam mode.

The characteristic for which today WIMS still stands out from the other currently available LMS is the built-in possibility of extensively using random parameters in the coding of the learning objects ${ }^{1}$ and the capability of interacting with software of many very different kinds. These two features combined allow for the creation of complex and engaging exercises. A well-designed use of random parameters enables WIMS to provide a virtually infinite set of copies of each single activity.

A first approach to WIMS. The simplest use of WIMS in mathematics is to let it act as a user-friendly web interface for asking for computations. For example, the tool

\footnotetext{
${ }^{1}$ Random parameters can also be used in the editing of documents.
} 


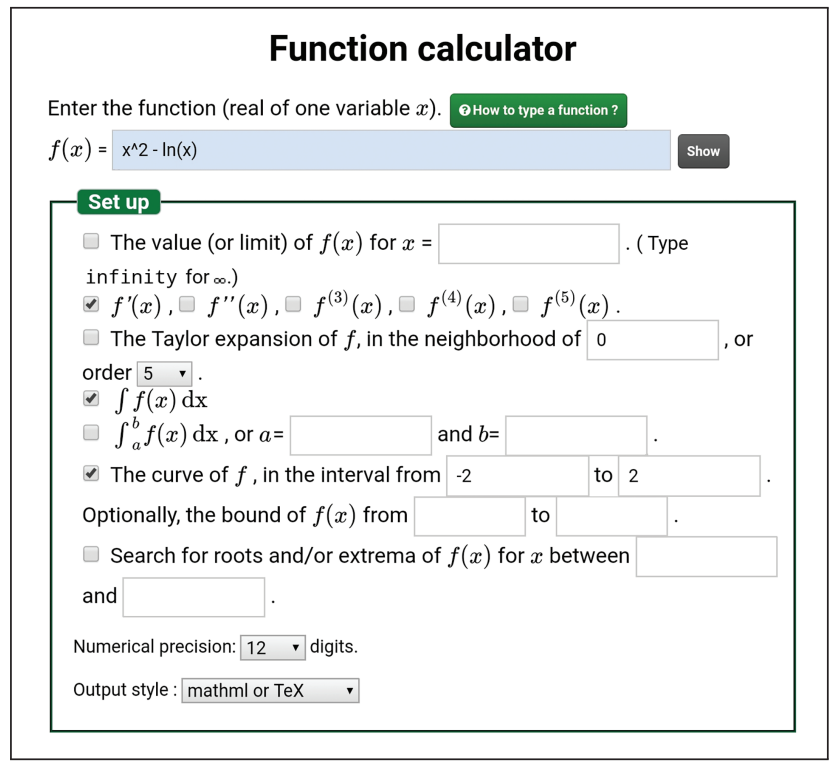

(a) Options

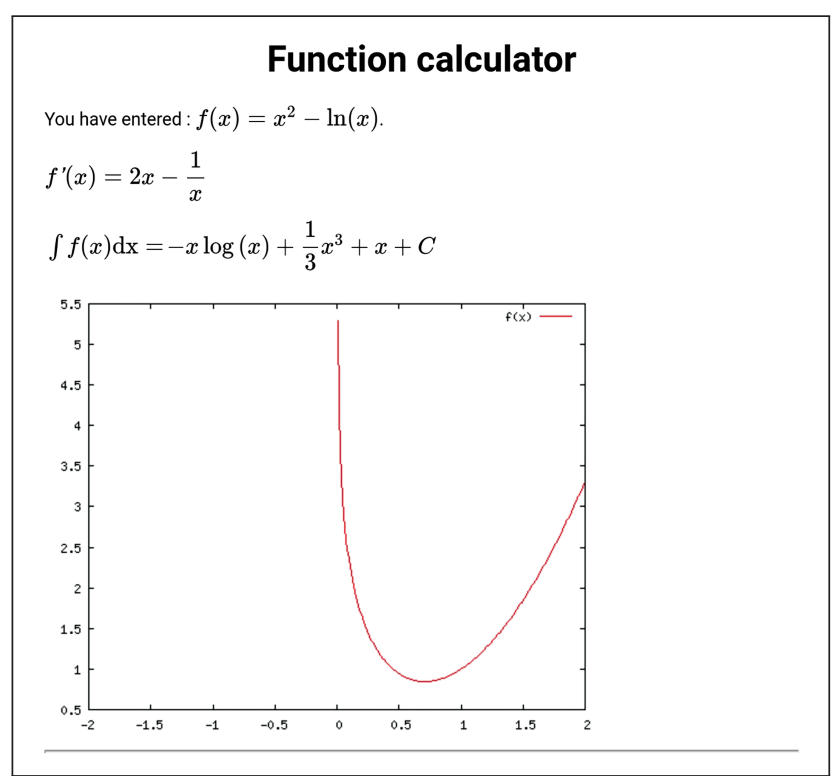

(b) WIMS' answer

Figure 1. WIMS tool: Function calculator

Function calculator interacts with open source programs Maxima, PARI/GP, and Gnuplot. The user inserts the query into the form shown in Figure 1a and gets the answer shown in Figure 1b: it is up to WIMS to choose the program most suitable for each task. ${ }^{2}$

If we focus on the more complex task of managing interactive activities and effectively analyzing user answers, one

\footnotetext{
${ }^{2}$ The tool Function calculator is freely available on any WIMS server, e.g., you can access and test it at https://wims.matapp.unimib.it/wims/wims .cgi?module=too7/analysis/function.en
}

of the strengths of WIMS is its ability to interact with representations and drawings. For example, the activity Triangular shoot ${ }^{3}$ allows the users to familiarize themselves with the centers of a triangle (barycenter, orthocenter, incenter, and circumcenter). Every time the exercise is selected, a different randomly generated triangle is proposed. In the easier version of the exercise, a triangle is given and the user has to click on one of the centers (see Figure 2a). The user's reply is evaluated and marked (see Figure 2b).

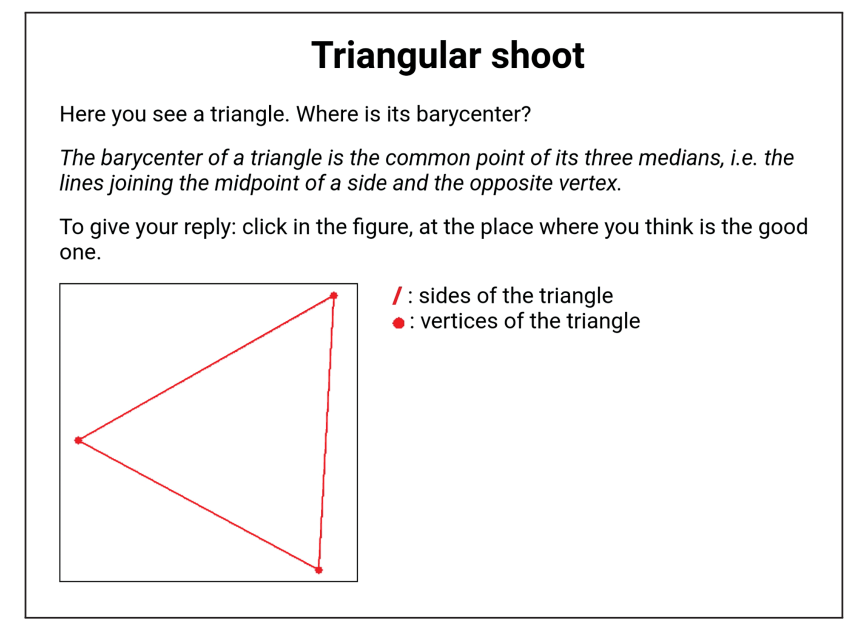

(a) Barycenter

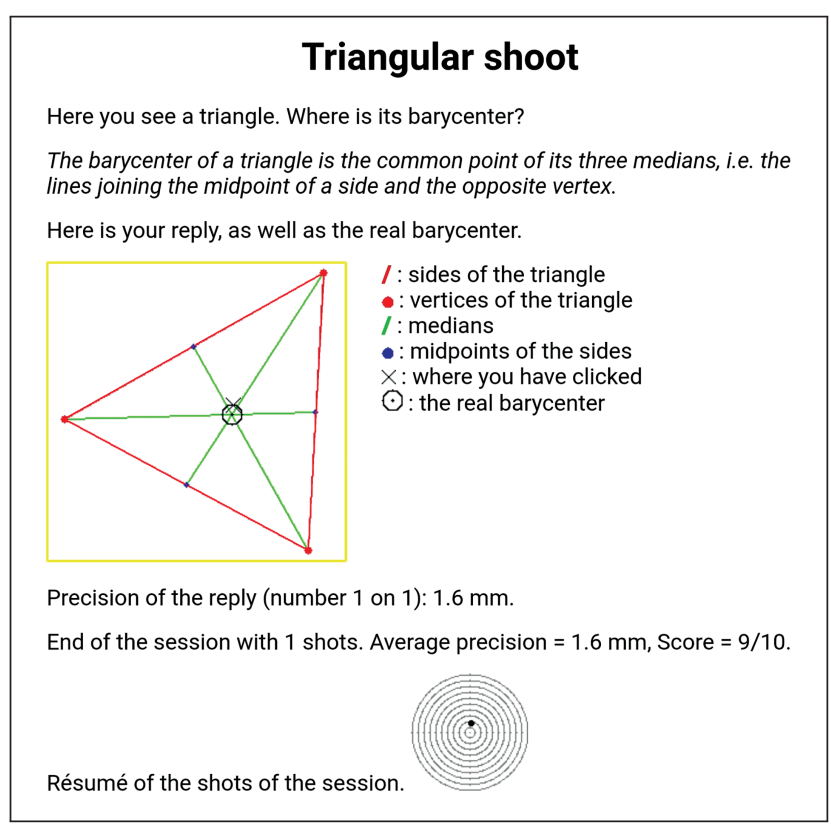

(b) Barycenter: analysis of the answer

Figure 2. Triangular shoot

https://wims . matapp. unimib. it/wims/wims . cgi ?modu Te=H4 /geometry/trishoot.en 


\section{EDUCATION}

\section{Triangular shoot}

Here you see one of the sides of a triangle, as well as its barycenter. Where is the third vertex of this triangle?

The barycenter of a triangle is the common point of its three medians, i.e. the lines joining the midpoint of a side and the opposite vertex.

To give your reply: click in the figure, at the place where you think is the good one.

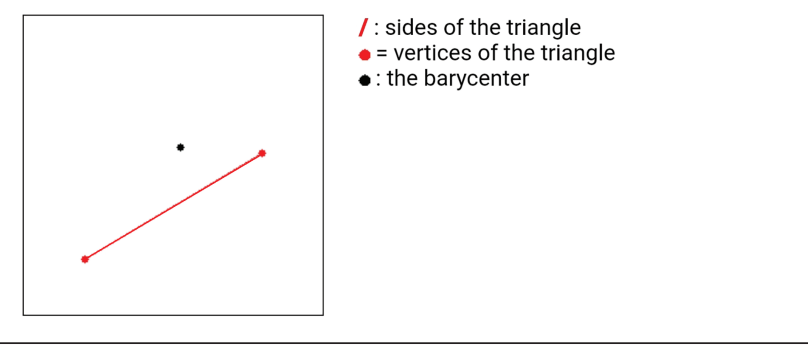

Figure 3. Triangular shoot: inverse problem

To give deeper experience with such concepts, Triangular shoot also includes the reverse problem (see Figure 3): given two vertices of the triangle and its barycenter, can you identify the missing vertex of the triangle?

The structure of WIMS. WIMS has a modular structure ("module" is the term used by WIMS for any of its units). A standard installation of the latest version of WIMS includes about 50 administrative modules and about 1800 learning modules. Whenever a new task is required, a new administrative module can be added to the system to provide such functionality. The learning modules include what WIMS calls "tools" (e.g., the already described Function calculator) as well as learning objects contributed by the teachers themselves (exercises, virtual classes, manuals, ...) designed according to their individual needs and then shared with the whole community.

As far as mathematics is concerned, WIMS is fully WTEX aware, and it can interact with the already mentioned Maxima, PARI/GP, and Gnuplot. It can also interact with GAP (e.g., see OEF permutation ${ }^{4}$ ), Povray (e.g., see Polyray ${ }^{5}$ ), Graphviz (e.g., see Graph drawing tool ${ }^{6}$ ), GeoGebra (e.g., see OEF GeoGebra ${ }^{7}$ ), Octave (e.g., see Statistical tables ${ }^{8}$ ). More recently the core of WIMS has been updated in order to interact with javascript applets. The drawing capabilities of JSXGraph and of HTML5 Canvas are now integrated into WIMS allowing much more interactivity from

${ }^{4}$ https://wims . matapp . unimib. it/wims/wims. cgi ?moduTe=U2 /aTgebra/oefperm.en

https://wims.matapp. unimib.it/wims/wims.cgi?module=too 7

Vgeometry/polyray.en

'https://wims . matapp. unimib. it/wims/wims.cgi?moduTe=tool /geometry/graphviz.fr

https://wims .matapp. unimib.it/wims/wims.cgi?modu7e=H1 /geometry/OEFgeog.fr

https://wims . matapp. unimib. it/wims/wims . cai?module=tool

/stat/table.fr the users. Other applets have been interfaced allowing for the creation of exercises in other subjects. For example, JSME and JSmol are used in exercises on molecular representations in the module Amino acids. ${ }^{9}$ JSmol is also used to draw polyhedra (see the online tool Convex Polyhedra $^{10}$ ).

All of WIMS' learning modules are classified by the authors via keywords according to the topics covered, and a search engine is provided. The ever expanding tree of keywords can be navigated via the Browse by subject ${ }^{11}$ module (see Figure 4). Classifications according to school levels (from preschool to university postdoc) are also provided.

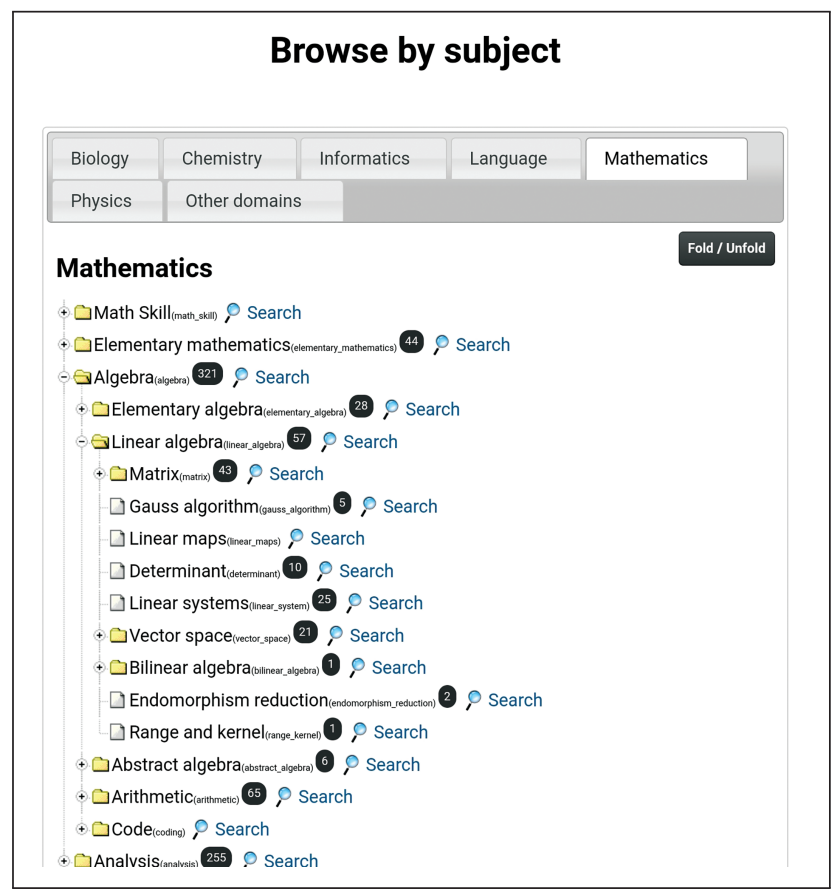

Figure 4. Browse by subject

\section{The Community}

Started as a one-person project, WIMS owes its growth to the large community that Xiao was able to gather around his work. The project was published as open source, giving open access to developers and to translators (the core of WIMS is available in French, English, Italian, Dutch, Chinese, Slovene, Spanish, and Catalan).

Xiao also called for the cooperation of teachers, promoting training seminars for them on the programming

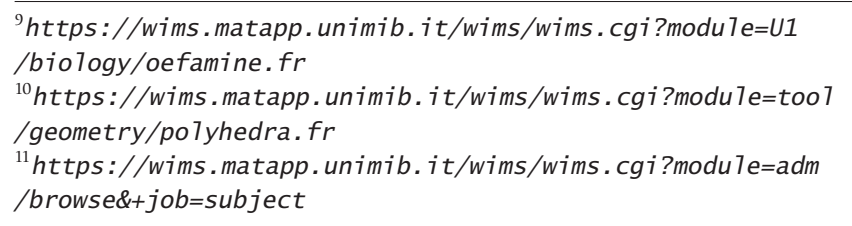


of exercises. From its very beginning, the development of WIMS relied on contacts between the developers and the community of teachers, who constantly report bugs and give ideas for improvements. Since 2006 periodical colloquia are held (e.g., see [7]), in order to discuss progresses in the use of WIMS and possible development strategies.

In July 2007, WIMSEDU ("Enseignants, développeurs et utilisateurs de WIMS") ${ }^{12}$ was founded, an association with the aim of strengthening this community: share skills, act as interlocutors between WIMS users and users of other software or institutions, discuss and influence the directions of its development by supporting the chosen projects, create a dynamic collaborative effort through its website, and identify, support, and inform about training actions. WIMSEDU carries on the tradition of the Colloques (Nice 2006, Nice 2007, Bordeaux 2010, Rennes 2012, Dunkerque 2014, Annecy 2016, Paris Orsay 2018, Amiens 2021) and the meeting of different expertises continues (mathematicians and nonmathematicians, developers and users, researchers and teachers from any educational level). In the colloquia user experiences are confronted, technical difficulties are discussed, and innovations are proposed. The association pursues its goal of acting as the glue for this community also by directly managing a server for distribution of learning modules through which the authors can share the exercises and documents they have designed for their students so that they are published on all of WIMS' servers. The actual development of the core of WIMS is carried out by a volunteer development team, whose activities are intertwined with WIMSEDU actions. Many of the team members are university staff, but no academic institution is directly involved, apart from hosting main servers in the WIMS network (servers generally used for institutional didactics). An important share of the work is still carried out by teachers (of any school level: from primary school teachers to university professors). The source code of the core of the system, in the open source spirit, is hosted by the French Public Interest Group RENATER ${ }^{13}$ which in this way provides essential support for WIMS. ${ }^{14}$

\section{WIMS for Mathematics Education}

WIMS can be used for mathematics teaching because it can provide students with many engaging exercises. There are many descriptions of experiences with WIMS for teaching (e.g., see [1], [2], [6], [9], [11], [12], [8], [3]), showing (at different school levels, in different class situations, in different subjects) that WIMS-based exercises, with automatic

\footnotetext{
${ }^{12}$ https://wimsedu.info

${ }^{13}$ French National Telecommunication Network for Technology, Education and Research

${ }^{14}$ The project page can be reached at the following url: https://sourcesup . renater.fr/projects/wimsdev/
}

marking, can motivate students and keep their attention alive. Moreover, WIMS can be used for exams, through special functions that guarantee an appropriate level of security and control.

Mathematical problem solving. We believe that problem solving à la Polya is a crucial step for a real understanding of mathematics. To gain a taste for mathematics, pupils should be led to actively work on a difficult problem and to develop a viable solution for such a problem, given the necessary amount of time [10]. The use of automated exercises might conflict with this idea. When given a large amount of similar exercises, students might get the idea that the goal of mathematics is to find the fastest strategy in order to get a computer-approved answer, a strategy that does not always correspond to a real understanding of the subject. This attitude is even stronger if WIMS exercises are used for exams. It is indeed a difficult equilibrium and particular effort must be put into the design and selection of the learning objects and on the monitoring of students' activity on these exercises. For example, WIMS proposes standard Number pyramids ${ }^{15}$ drills for practicing sums (e.g., at a primary school level) as the one shown in Figure 5a. If compared to a corresponding paper and pencil version of this exercise, the advantage of this computer-based version is that the student has immediate feedback and can repeat the exercise as many times as wanted. It is easy to predict, and in fact we can observe the phenomenon, that this exercise can lead the students to develop improper strategies: after a few tries the students can understand that there is actually no need to calculate any sum, and that a computerapproved solution of the exercise can be obtained very quickly just by putting the biggest number above and the two smaller numbers below. This way of finding the answer for the exercise can be considered wrong if the aim of the teacher is to have students work with computation of sums. The teacher should monitor students' work and, if needed, should act so as to avoid students relying on this unwanted strategy, and lead them to proceed a step forward to tackle more advanced tasks. A simple way to gain this is to switch to a version of the same exercise which proposes more choices for the numbers to be dragged into the pyramid, as the one shown in Figure 5b. The presence of an extra number to be set aside forces the user to actually do the sums (or to find a more creative strategy!). The example in Figure 5b also allows us to show that in WIMS you can design exercises with multiple correct solutions, thus countering the formation of one of the most deleterious students' belief: "Mathematics problems have one and only one right answer" (cf. [13, p. 359]).

19 https://wims. matapp. unimib. it/wims/wims. cgi ?modu7e=HI TaTgebra/addfig.en 


\section{EDUCATION}

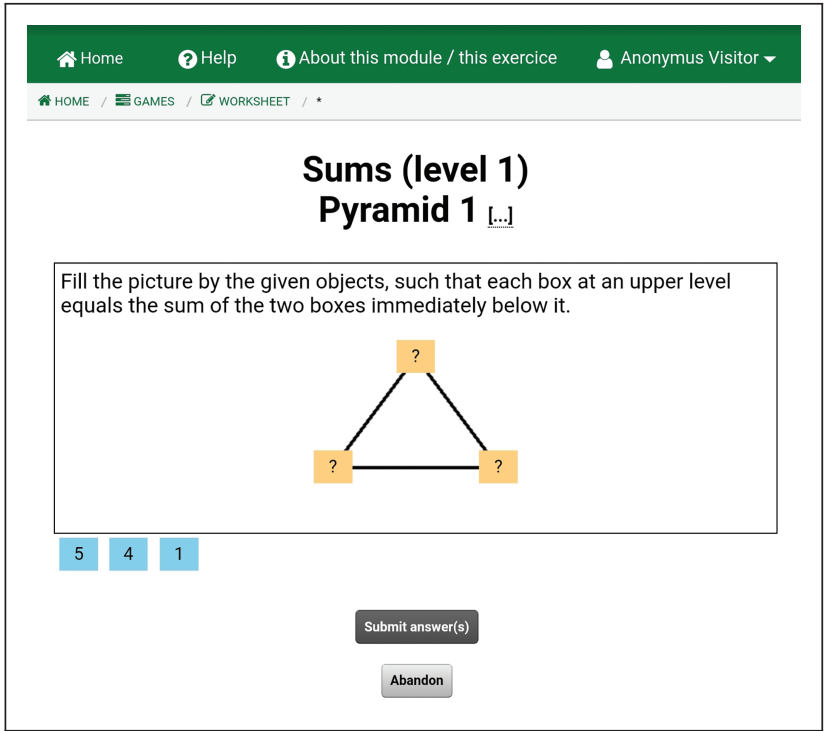

(a) Number pyramids I

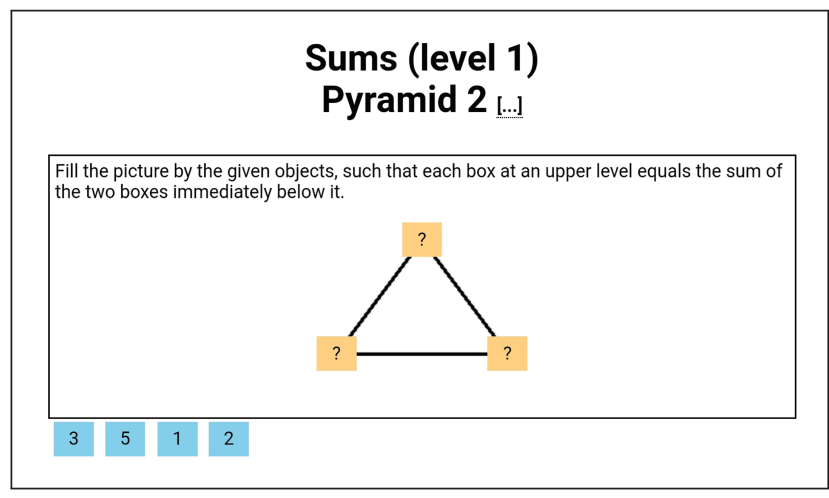

(b) Number pyramids II

Figure 5. Number pyramids

Furthermore, from a problem-solving perspective, automatically presenting a full solution for the exercise as soon as the student makes a mistake can be counterproductive, as this could prevent the actual understanding of the errors and interfere with the pursuit of an autonomous solution. WIMS allows for the creation of feedback that provides alerts for classical errors or hints to help students to persevere in their search. For example the module Probability distribution plots ${ }^{16}$ contains an activity that shows how feedback can indicate to the student an expected property that is not satisfied by his/her answer (see Figure 6). Teachers that use WIMS with their students can interact with the exercises's author and suggest suitable feedback.

Mathematicians' point of view. Mathematicians can contribute to the development of WIMS by pointing out which

${ }^{16}$ https://wims. matapp. unimib. it/wims/wims.cgi?modu7e=U1 proba/oefgraphlaw.fr

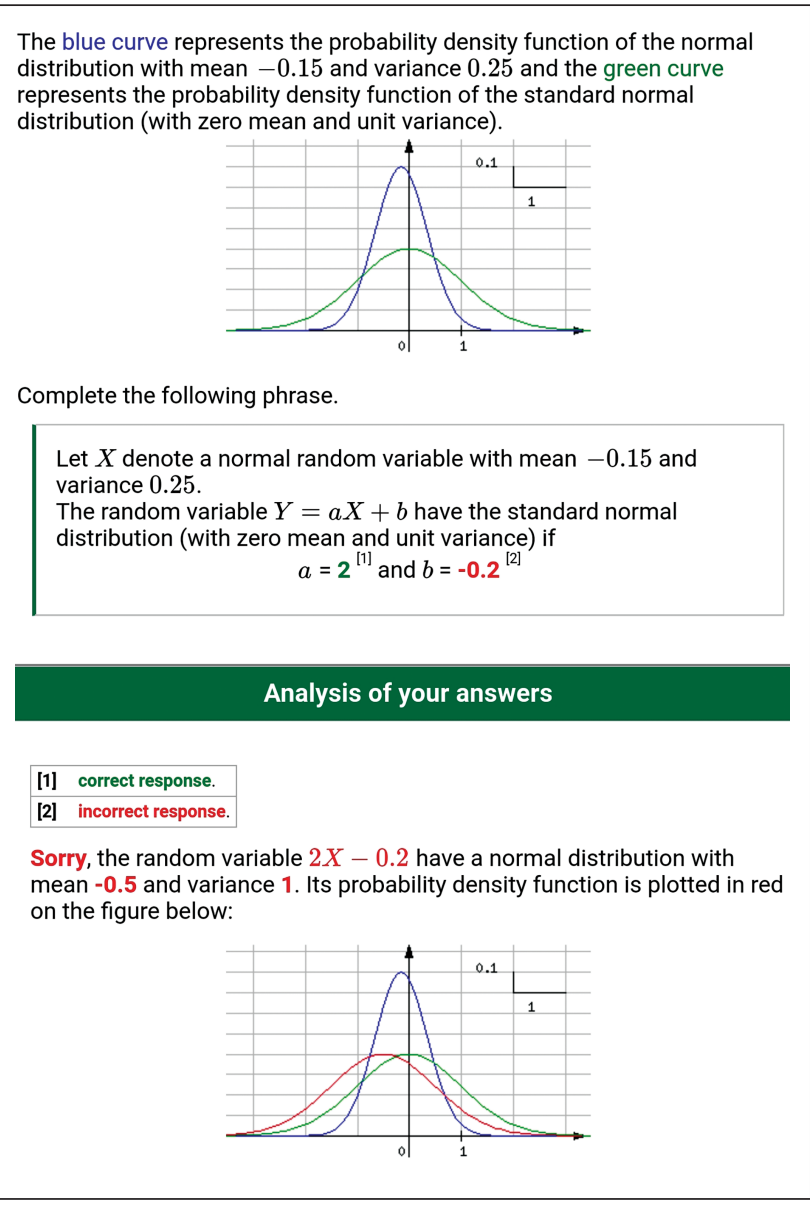

Figure 6. Statement and feedback in exercise A standard normal random variable

aspects of the discipline the learning objects should focus on. Going back to Triangular shoot we can notice that the author of the exercise (a mathematician!) did not want to test users on the question "what is the barycenter of a triangle," as the definition is right in the statement shown to the user (as you can see in Figure 2a). Rather, the aim of the exercise is to use the definition and actively manipulate such a notion. Too often students are led to believe that most of mathematics consists of pure memorization of facts, and do not manage to get any real insight into the different facets of each concept.

A problem should be the means to lead students to grasp the theory underneath it. An example in this direction is the series of activities on symmetry that are proposed: these exercises are built on different levels, to gradually lead learners to tackle more and more complex concepts. For example, in the module OEF Rosettes ${ }^{17}$ you can start (level A) with the simple task of tracing any

17 https://wims. matapp. unimib. it/wims/wims. cai ?module=U2 geometry/oefrosoni.it 
symmetry axis of a given figure, and later you can proceed to the more complex task (level D, shown in Figure 7) of finding the two symmetry axes that generate the whole $* n$. kaleidoscopes rosette pattern (e.g., see [5]).

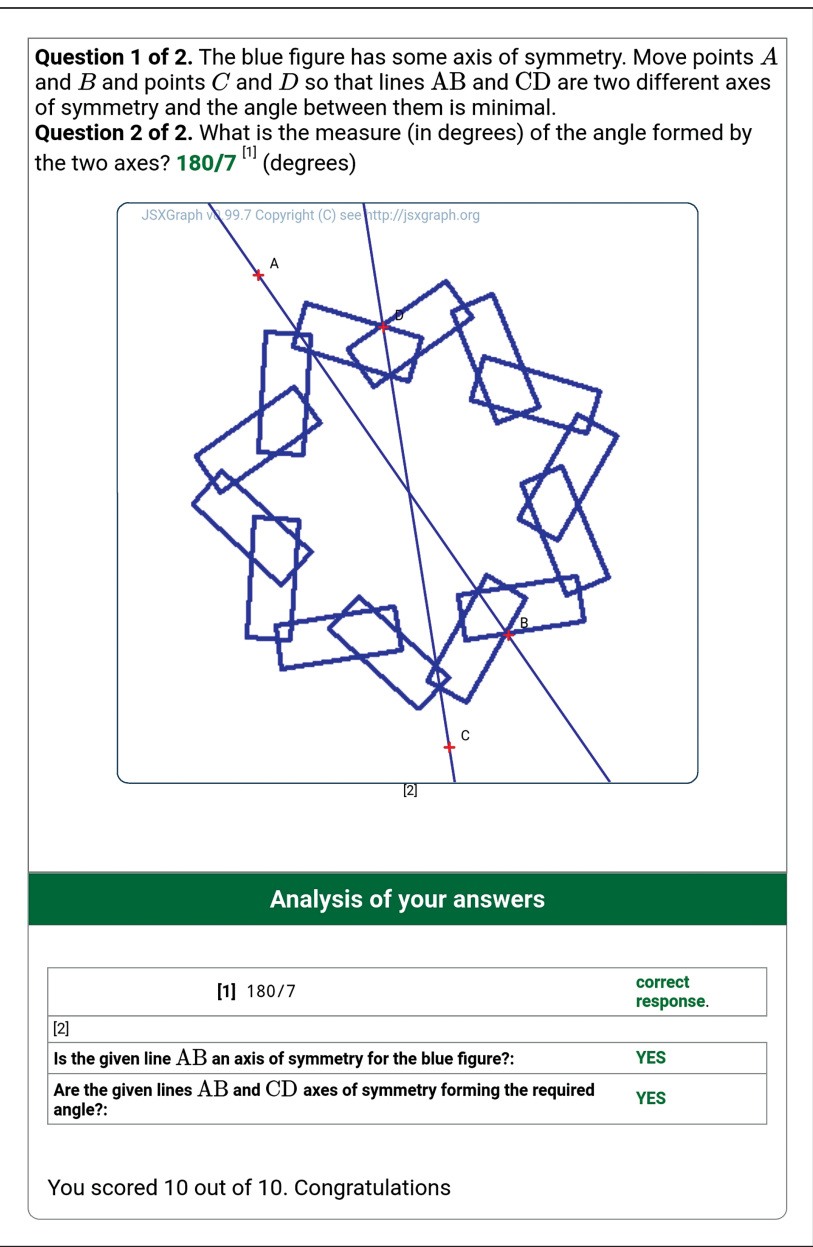

Figure 7. Symmetry axes (level D)

The idea of manipulating mathematical objects can be exploited in various fields of mathematics. The module OEF Graphics study of differential equations or simple differential systems ${ }^{18}$ contains an activity in which the user can interact with the solution curves of a differential system, in order to gain information about its isoclines (see Figure 8). A similar manipulation can be useful in other domains, such as probability, where many results are counterintuitive for beginners. Exercises in which simulated data and probability distributions are presented can help students to have a better understanding of what kind of events may appear frequently by chance and what a probability distribution represents. For example, in the

18 https://wims . matapp. unimib. it/wims/wims. cai ?modu7e=U2 /ana7ysis/oefgrapheqdiff.fr

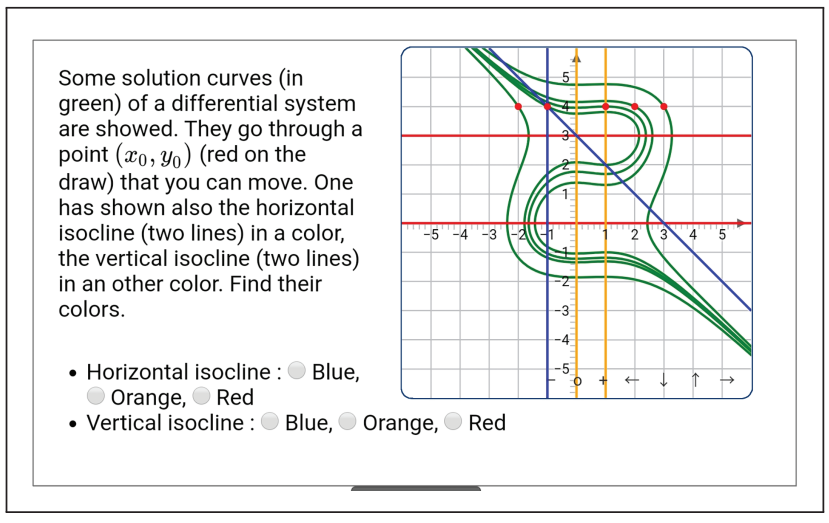

(a) Statement

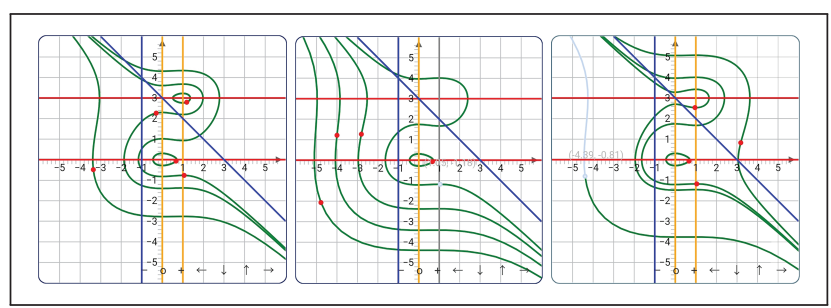

(b) Manipulations by the user

Figure 8. Isoclines

exercise Poisson distribution of the module Point estimation in statistics, ${ }^{19}$ the outcome of a Poisson point process simulation on a square is drawn. The aim of the exercise is to find the empirical measure of the number of points in each cell of the given Cartesian grid and to estimate the intensity of this process (see Figure 9). Once the student has given his/her answer, the relative frequencies of the number of points in each cell and the probability mass function of the Poisson distribution with the estimated parameter are shown in the same grouped bar chart through feedback. The fact that the two sets of bars can be quite different surprises some students.

Traditional problems can become a source of inspiration for creating learning objects. For example, the problem of studying "lattice polygons," that is, plane polygons with vertices with integer coordinates, has been widely explored and can give rise to nice results such as Pick's theorem. The module OEF Polygons on graph paper ${ }^{20}$ offers a series of exercises asking for the tracing of triangles and quadrilaterals with integer coordinates vertices. The rationale behind the module is that exploring the variety of existing examples of polygons opens the mind to a more advanced geometric vision, so the aim of the exercises is to

\footnotetext{
19 https://wims . matapp . unimib. it/wims/wims. cgi ?modu7e=U1 /stat/oefestimation.fr

${ }^{24}$ https://wims. matapp. unimib. it/wims/wims. cgi?module=E4 /geometry/oefpo7yqq.it
} 
Positions of bees in a flowering rapeseed field are drawn in red. The field is divided into 25 plots of $5 \mathrm{~m}$ each. The number of bees observed in each plot is given for clarity.

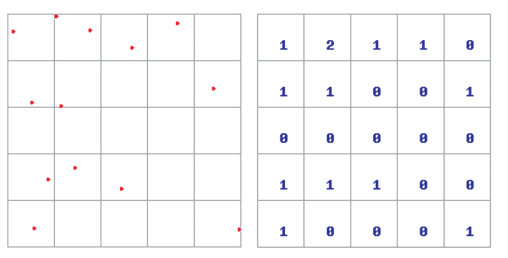

These numbers are modelled as the observed values of independent Poisson-distributed random variables $X_{1}, \ldots, X_{25}$

1. Complete the relative frequency table of these 25 data:

\begin{tabular}{|c|c|c|c|}
\hline number of bees in a plot & $\mathbf{0}$ & $\mathbf{1}$ & $\mathbf{2}$ \\
\hline relative frequency & 0.52 & 0.44 & 0.04 \\
\hline
\end{tabular}

Good answer! Here is the relative frequency histogram:

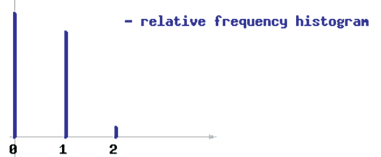

2. What is the value of the empirical mean of these data? $0.52^{[1]}$

Analysis of your answers

relative frequency for $0=0.52$ correct response.

relative frequency for $1=0.44$ correct response.

relative frequency for $2=0.04$ correct response.

[1] 0.52 correct response.

In the figure below, the relative frequency histogram can be compared with the probability mass function of the Poisson distribution with expectation 0.52 .

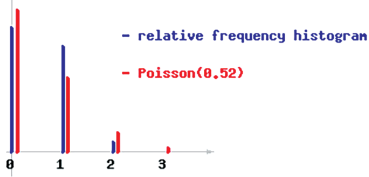

Figure 9. A statement of exercise Poisson distribution with feedback when the student's answers are correct.

stimulate the construction of nonstereotyped examples, by asking questions such as:

- trace a quadrilateral with all equal sides and with no sides laid on the grid,

- trace a quadrilateral with perpendicular and equal diagonals and with sides all of different lengths,

- trace a quadrilateral with exactly two right angles and with no sides laid on the grid.

It might be interesting to point out that users' replies for such constructions are evaluated via basic $2 \mathrm{D}$ vector geometry notions, using PARI/GP: the coding of the exercise itself becomes a playground to see applied mathematics at work.

A computer-based learning system can be used to introduce standard algorithms. In WIMS you can find, for instance, the module Parmsys ${ }^{21}$ that leads the user through the steps of Gauss' elimination method for reducing a linear system with parameters in order to determine whether it is solvable or not (Figure 10). Activities of this kind, in which the task of actually making all the computations is left to the computer, allows the students to focus on the problem itself: which are the most convenient steps to get to the triangular form for the system? Once the algorithm

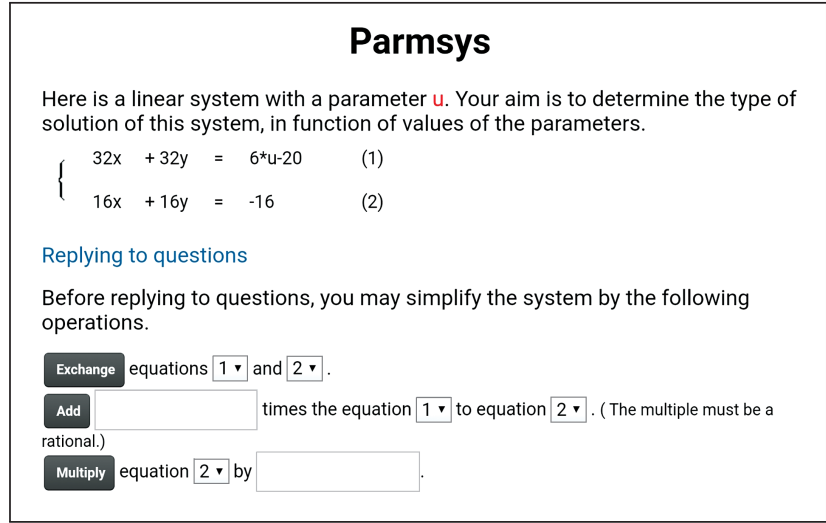

Figure 10. Gauss' algorithm in linear algebra

has been assimilated, it is possible to propose modeling exercises for different applications. The module OEF linear systems ${ }^{22}$ contains the exercise Alloy of 3 metals in which students are explicitly invited to use the tool Linear solver $^{23}$ so that they do not need to spend too much time on computations, but they can concentrate on the main difficulty of this type of exercises which is indeed to define the linear system they have to solve to answer the questions.

Finally, and this time we exit the domain of mathematics and provide an example in biology, even more interactive activities can be built. In the module Identification of animal tissue ${ }^{24}$ a picture of a biological tissue to be identified is shown (see Figure 11a) and the user is guided through a standard analysis protocol with ad hoc questions. Users' answers are checked by the system one by one (see Figure 11b), and, in case of errors, intermediate questions can be added to allow for the student to rectify any mistake or to follow an alternative approach. Only after every single reply has been given feedback is the final question asked: "What is the tissue category?" This

21 https://wims.matapp. unimib. it/wims/wims. cgi?modu 7e=U1 Va7gebra/parmsys.en

${ }^{22}$ https ://wims . matapp. unimib. it/wims/wims . cq7 ?module=H6 Talgebra/oeftinsys.en

${ }^{23}$ https://wims . matapp. unimib. it/wims/wims. cgi?module=tool /7inear/7insolver.en

${ }^{24}$ https://wims . matapp . unimib. it/wims/wims. cgi?modu $7 e=U 1$ Vhistology/identanima7.fr 
example also allows us to see the idea of "data modules" at work: the set of images of tissues to be identified is available to be shared with any other learning module on similar subjects.

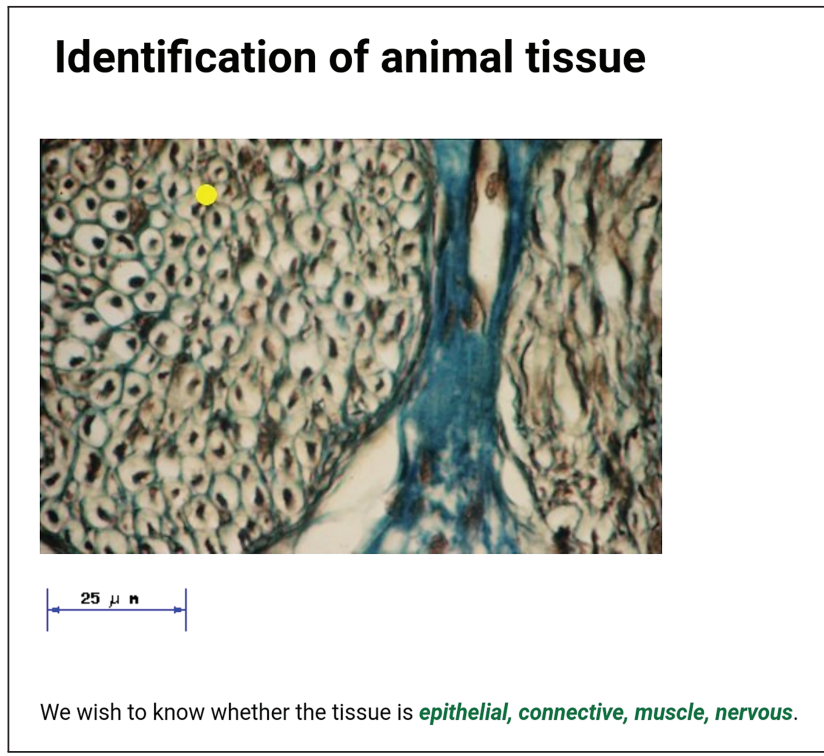

(a) Animal tissue I

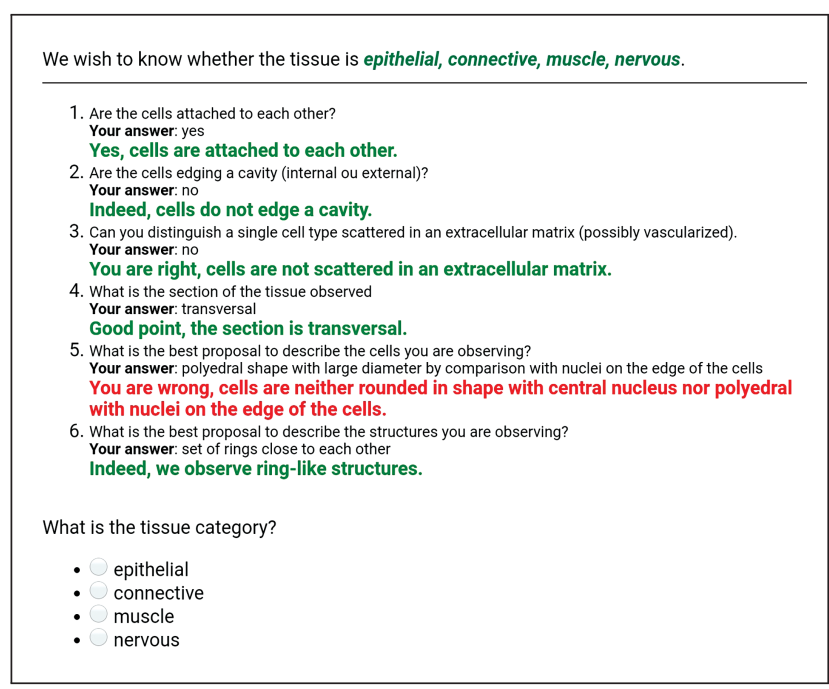

(b) Animal tissue II

Figure 11. Example in biology

Teachers' point of view. Through any WIMS server, teachers have access to a large variety of learning objects, on many different subjects, beyond mathematics. Available "Open classes" provide models for ready-to-use courses on various topics, so that teachers, with just one click, can create their own copy of the class and have access to all of the LMS functions available (selecting exercises, monitoring students' progresses, grading, ...). Teachers have thus the option to use already existing materials as is, but they also have the option to adapt anything to their own teaching style. Moreover, they can create their own exercises. WIMS provides a somehow simplified programming language, called OEF ("Online Exercise Format") that allows for the use of all the advanced features of WIMS and it is fully documented on line. WIMS also provides tools to create standard exercises, like multiplechoice questions or "true or false" or "fill in the blanks" and so on. Each WIMS module in the distribution is open source, so the teacher is encouraged to look inside any exercise and have an understanding of how it really works, not merely accepting mysterious turnkey packages.

Teachers have the possibility to select among many different configurations for each single instance of any OEF exercise: if the author of the exercise provides a hint or a solution, it is up to the teacher to decide whether the hint or solution is actually shown to the students. Teachers can write alternative hints or solutions themselves to be shown to the students, using WIMS documents (in this case with no need to edit the exercise code). Teachers can also set up the exercise so that in case of errors the students have to go through the very same exercise again: as WIMS exercises are highly randomized, usually you can expect a different version of the exercise any time you open it; with this option students have the chance to revise their resolution by themselves and empower their own self-directed learning skills.

Finally, the fact that WIMS covers many subjects apart from mathematics allows teachers to have everything in one place and favors interdisciplinary links. For example, it allows for the connection of the idea of the barycenter of a triangle seen in Figure 2a to the problem of finding the center of gravity of a system of weighted objects as in the module Gravity shoot ${ }^{25}$ shown in Figure 12.

\section{Conclusion}

We have tried to highlight the most significant aspects of the WIMS system and to bring our experience on the potential of using new technologies in teaching. We hope to have encouraged the reader to visit the site, not letting themselves be restrained by a sometimes bit too intricate graphical aspect, but willing to really explore the numerous learning objects available. We also hope to have encouraged participation in our community: there is a lot of work to be done in order to keep the WIMS system active and make the already available materials better usable. A special effort in this direction concerns the translation into other languages of the huge resource of learning modules

${ }^{25}$ https://wims . matapp. unimib. it/wims/wims . cgi ?modu 7e=H5 Vgeometry/gravshoot.en 


\section{Gravity shoot}

We have 5 objects with different weights in the plane. The following figure shows the positions of these 5 objects. Where is the gravity center of the set of these 5 objects?

To give your reply: click in the figure, at the place where you think is the good one.

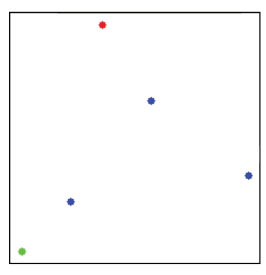

= object of weight $=$ object of weight 2 $=$ object of weight 3

Figure 12. Gravity shoot

existing in French. We also feel the need for building a better database of available resources and of improving examples of turnkey "Open classes," and, again, to translate modules and classes into many more languages. Work has to be done to guarantee the operativity of modules in spite of software obsolescence and in order to take advantage of developing technologies: Xiao's modules, dating back to 1998 , are still fully functional. And we constantly need to plan the future development of the system.

Once more we recall the questions raised in [4], that still remain open:

- Which are the most effective interactive activities, and can the strengths of WIMS help teachers offer such activities to their students?

- Can WIMS be used to evaluate students' achievements?

- Can WIMS be used to build personalized learning paths?

- How to develop the technological knowledge of teachers in order to enable them to exploit the full potential of WIMS?

- Can the capabilities of WIMS be embedded into other LMS, such as, e.g., Moodle?

Any contributions from researchers in the subjects covered, from computer scientists, and from researchers in education can support the development of WIMS in a direction that improves its effectiveness in the communication of science and in teaching.

\section{References}

[1] Claire Cazes, Ghislaine Gueudet, Magali Hersant, and Fabrice Vandebrouck, Using e-exercised bases in mathematics: case studies at university, International Journal of Computers for Mathematics Learning 11 (2006), 327-350.

[2] Marina Cazzola, WIMS all'Università di Milano-Bicocca, TD Tecnologie Didattiche 19 (2011), no. 3, 170-175.
[3] Marina Cazzola, Play with maths: Mathematical games through WIMS, Iceri2019 proceedings, 2019, pp. 22972305.

[4] Marina Cazzola, Bernadette Perrin-Riou, and Eric Reyssat, WIMS: a WWW interactive multipurpose server, In proceedings of ICTMT11, 2013.

[5] John H. Conway, Heidi Burgiel, and Chaim GoodmanStrauss, The symmetries of things, A K Peters, Ltd., Wellesley, MA, 2008. MR2410150

[6] Csilla Ducrocq, Lutilisation des dictionnaires et des collections de documents audio libres en ligne pour créer des exercices en langue sur la plate forme d'enseignement numérique WIMS, Recherche et pratiques pédagogiques en langues de spécialité. Cahiers de l'Apliut 29 (2010), no. 1, 172-177.

[7] Fabrice Guérimand, Bernadette Perrin-Riou, and Eric Reyssat (eds.), WIMS, échanges sur les pratiques et formation à l'outil, Crdp de l'Académie de Nice, 2e colloque international du 9 au 11 mai 2007, Université de Nice SophiaAntipolis, 2008.

[8] Magdalena Kobylanski, WIMS: Innovative pedagogy with 21 year old interactive exercise software, Technology in mathematics teaching, 2019, pp. 123-144.

[9] Boštjan Kuzman, Interactive mathematics in a WIMS virtual classroom (Interaktivna matematika v spletni učilnici WIMS), Mednarodna konferenca splet izobraževanja in raziskovanja $z$ ikt, 2007, pp. 258-261.

[10] George Pólya, Mathematical discovery, John Wiley \& Sons, Inc., New York, 1981. On understanding, learning, and teaching problem solving; Reprint in one volume; With a foreword by Peter Hilton; With a bibliography by Gerald Alexanderson; With an index by Jean Pedersen. MR624067

[11] Marie-Joelle Ramage and Bernadette Perrin-Riou, La technologie au service de pratiques d'apprentissage différenciées: La plateforme WIMS, utilisation en premier cycle universitaire, Actes du colloque TICE 2004, 2004.

[12] Eric Reyssat, Enseigner avec WIMS : exercices et documents interactifs à variation aléatoire, Actes of CETSIS 2013, 2013.

[13] Alan H. Schoenfeld, Learning to think mathematically: Problem solving, metacognition, and sense-making in mathematics, Handbook for research on mathematics teaching and learning, 1992, pp. 334-370.

[14] Gang Xiao, WIMS: An Interactive Mathematics Server, Journal of Online Mathematics and its Applications 1 (2001), no. 1. Retrieved December 9, 2019, from https://www.maa.org/press/periodicals /1oci/joma/wims-an-interactive-mathematics -server 


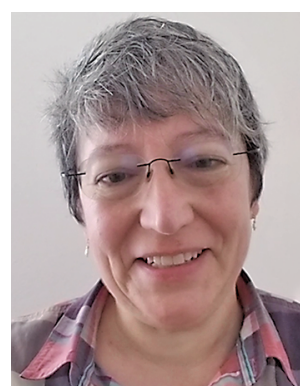

Marina Cazzola

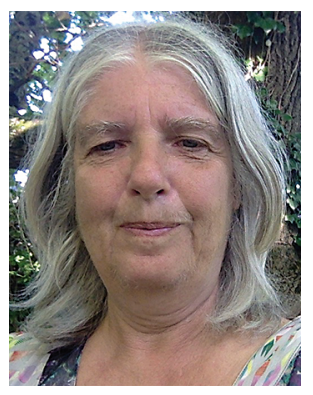

Bernadette

Perrin-Riou

\section{Credits}

Figures $1-10,11 b$, and 12 are courtesy of the authors.

Figure $11 \mathrm{a}$ is courtesy of Florence Mougel-Imbert.

Photo of Marina Cazzola is courtesy of Marina Cazzola.

Photo of Sophie Lemaire is courtesy of Sophie Lemaire.

Photo of Bernadette Perrin-Riou is courtesy of Bernadette Perrin-Riou.

\section{Combinatorial Algebraic Geometry}

\section{February 1 - May 7, 2021}

ORGANIZING COMMITTEE

Anders Buch, Rutgers University

Melody Chan, Brown University

June Huh, Princeton University

Thomas Lam, University of Michigan

Leonardo Mihalcea, Virginia Polytechnic Institute

Sam Payne, University of Texas at Austin

Lauren Williams, Harvard University

\section{PROGRAM DESCRIPTION}

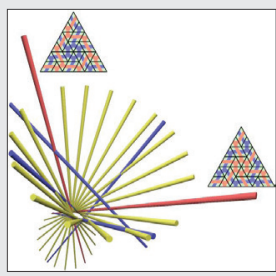

Research in combinatorial algebraic geometry utilizes combinatorial techniques to answer questions about geometry. It also uses geometric methods to provide powerful tools for studying combinatorial objects.

Much research in combinatorial algebraic geometry relies on mathematical software to explore and enumerate combinatorial structures and compute geometric invariants. Writing the required programs is a considerable part of many research projects. The development of new mathematics software is therefore prioritized in the program.

This program will bring together experts in both pure and applied parts of mathematics as well mathematical programmers, all working at the confluence of discrete mathematics and algebraic geometry, with the aim of creating an environment conducive to interdisciplinary collaboration.

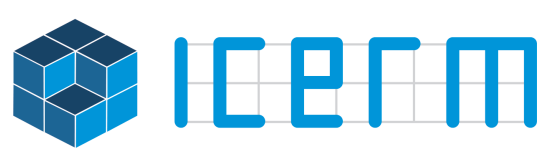

Institute for Computational and Experimental Research in Mathematics

Proposals being accepted: Semester Program

Topical/Hot Topics Workshops Small Group Research Program Summer Undergrad Program

ICERM is a National Science Foundation Mathematics Institute at Brown University in Providence, RI.
Appications being accepted: Semester Program or Workshop Postdoctoral Fellowship

Sponsorships being accepted: Academic or Corporate

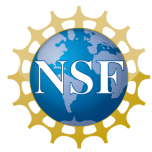

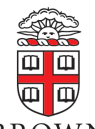

BROWN

\section{icerm.brown.edu}

